\title{
Increased levels of serum osteoprotegerin in hypothyroid patients and its normalization with restoration of normal thyroid function
}

Toshiki Nagasaki, Masaaki Inaba, Shuichi Jono, Yoshikazu Hiura, Hideki Tahara, Kumi Shirakawa, Naoyoshi Onoda ${ }^{1}$, Tetsuro Ishikawa ${ }^{1}$, Eiji Ishimura and Yoshiki Nishizawa

Department of Metabolism, Endocrinology and Molecular Medicine and ${ }^{1}$ First Department of Surgery, Osaka City University Graduate School of Medicine, 1-4-3, Asahi-machi, Abeno-ku, Osaka 545-8585, Japan

(Correspondence should be addressed to Masaaki Inaba; Email: inaba-m@med.osaka-cu.ac.jp)

\begin{abstract}
Hypothyroidism is associated with increased morbidity from cardiovascular disease, and an increase in serum osteoprotegerin (OPG) has recently been reported to be associated with the severity of coronary heart disease and cardiovascular mortality. The present study was designed to examine whether hypothyroidism causes an increase in serum OPG, and to determine whether levothyroxine (L-T4) replacement therapy might suppress serum OPG levels in hypothyroid patients. Fifty-three hypothyroid patients with chronic thyroiditis and age- and sex-matched normal control subjects were examined for the levels of serum OPG and plasma von Willebrand factor (vWF), a vascular injury marker. Thirty-seven of the hypothyroid patients were further monitored for changes in these markers during 1 year in a euthyroid state induced by L-T4 replacement therapy. Baseline OPG was significantly higher in hypothyroid patients than in normal controls $(4.51 \pm 0.50$ vs $3.72 \pm 0.23 \mathrm{pmol} / \mathrm{l}$ (mean \pm S.E.); $P=0.0182$ ). In multivariate analysis, baseline OPG was significantly associated with baseline levels of TSH $(r=0.280, P=0.0162)$ and vWF $(r=0.626$, $P<0.0001)$. During one year of L-T4 replacement therapy, hypothyroid patients showed a significant decrease in OPG levels from $4.35 \pm 0.51$ to $3.48 \pm 0.26 \mathrm{pmol} / \mathrm{l}(P=0.0166)$, a level comparable to normal controls. The change in serum OPG levels during L-T4 replacement therapy was significantly and independently associated in a negative fashion with baseline vWF $(r=-0.503, P=0.0014)$. This study suggested that the severity of hypothyroidism and vascular injury might have important independent roles in increasing the serum OPG level in hypothyroid patients. Furthermore, it was demonstrated that a sustained euthyroid state might have the potential to decrease the serum OPG level in hypothyroid patients and that the degree of vascular injury in the hypothyroid state is independently associated with a decrease in serum OPG during a 1-year normalization of thyroid function.
\end{abstract}

European Journal of Endocrinology 152 347-353

\section{Introduction}

Osteoprotegerin (OPG), a member of the tumor necrosis factor receptor family, has been identified as a regulator of bone resorption (1). OPG is produced by a variety of tissues besides bone, such as the cardiovascular system (vascular cells including coronary smooth muscle cells and endothelial cells in vitro (2)), lung, kidney and immune cells $(1,3)$. It has been shown that OPGdeficient mice develop severe osteoporosis and medial calcification of the aorta and renal arteries (4), and that OPG immunoreactivity can be demonstrated in early atherosclerotic lesions in human tissues (5). A recent clinical study reported an increase in serum OPG as a risk factor for cardiovascular mortality (6), and our recent report (7) demonstrated a significant association between increased serum OPG and the presence and severity of coronary heart disease.
Hypothyroidism, even in its latent form, is associated with increased morbidity from cardiovascular disease $(8,9)$. We recently reported that intima-media thickness (IMT) in the common carotid artery, a good predictor for cardiovascular events $(10,11)$, is increased in hypothyroid patients, and that increased IMT normalized after one year of levothyroxine (L-T4) replacement therapy (12). Accelerated atherosclerosis in hypothyroidism is accounted for by multiple mechanisms, including hyperlipidemia (13), hypercoagulable state (14), impaired endothelium-dependent vasodilation (15) and obesity (16). However, since there is no data on serum OPG levels in hypothyroidism, it is of importance to assess the possible involvement of OPG in the increased arterial thickening in hypothyroidism.

This background scenario prompted us to examine (i) whether serum OPG might be increased in hypothyroid patients, (ii) whether serum OPG might decrease after 
normalization of thyroid function by L-T4 replacement therapy, and (iii) which of a number of clinical variables, including blood pressure, body weight, thyroid hormone level, and the level of von Willebrand factor (vWF), a marker of vascular injury (17-21), is most important in influencing serum OPG in hypothyroid patients and its changes during L-T4 replacement therapy.

\section{Patients and methods}

\section{Patients and study design}

The present study was designed as an open prospective study. Written informed consent was obtained from each patient, and the study was approved by the ethics committee of Osaka City University Hospital. Fifty-three hypothyroid patients (10 males, 43 females) were enrolled in the study, among whom 37 patients ( 6 males, 31 females) were studied before and after undergoing L-T4 replacement therapy. The diagnosis of hypothyroidism was established on the basis of suppression of serum free thyroxine (FT4) and free triiodothyronine (FT3) to below the normal lower limit, and elevation of serum thyroid-stimulating hormone (TSH) to above the normal upper limit. The etiology of all hypothyroid patients was chronic thyroiditis as evidenced by the presence of firm diffuse goiter with a positive thyroid test and/or microsome test. The dose of L-T4 administered was adapted individually to maintain serum FT4, FT3 and TSH levels within their respective normal ranges. Biochemical markers, including serum OPG and plasma vWF, were measured in all patients before and at least 1 year (12-14 months) after normalization of thyroid function. Fifty-three healthy control subjects were enrolled from the participants in a local health-check program conducted at the Osaka City University Hospital. They had neither a past history of thyroid disease nor goiter. To avoid complications from other factors well known to affect atherosclerosis, patients meeting either of the following criteria were excluded from the study: (i) patients taking medication known to affect atherosclerosis, such as anti-hypertensive agents, lipid-lowering drugs, and anti-platelet drugs, and (ii) patients receiving other hormone replacement therapy.

\section{Serum parameters}

Blood was withdrawn just after an overnight fast, and vWF levels in plasma were measured as previously described (20-22). Total cholesterol, triglyceride and high-density lipoprotein (HDL)-cholesterol levels were determined using an autoanalyzer. The level of lowdensity lipoprotein (LDL)-cholesterol was calculated according to the formula given by Friedewald (23). FT4, FT3 and TSH were measured using commercially available kits (Ortho-Clinical Diagnostics, Amersham,
Bucks, UK), using a one-step nonradioactive immunoassay for FT4 in the dedicated Amerlite enhanced luminescence assay system (24). Briefly, a monoclonal antibody to $\mathrm{T} 4$, conjugated with horseradish peroxidase (HRP; EC 1.11.1.7), competes for binding with FT4 or with a conjugate of a protein and levotriiodothyronine (L-T3) immobilized onto the surface of the assay microwells. The signal is generated by antibodyHRP bound to the protein-L-T3 conjugate, with luminol as substrate.

OPG levels were determined in serum samples which had been kept at $-80^{\circ} \mathrm{C}$, using a sandwich ELISA (Cosmo Bio, Tokyo, Japan) as previously described (25). A monoclonal antibody against human OPG, designated as clone OI-19, was used to separate OPG from serum. The OPG was then detected with a peroxidase-labeled antihuman OPG monoclonal antibody, designated as clone OI-4, and a tetramethylbenzidine substrate. All samples were measured in duplicate and the results were averaged. The detection limit of this assay system was $0.03 \mathrm{ng} / \mathrm{ml}$, and the intra- and interassay coefficients of variation were $<3.2 \%$ and $<5.4 \%$ respectively $(25)$.

\section{Statistical analysis}

Data are expressed as means \pm s.E. unless otherwise indicated. Statistical analysis was performed with the StatView V system (Abacus Concepts, Berkeley, CA, USA) on an Apple computer. Differences in basal values between hypothyroid patients and normal controls and the difference in each parameter before and after treatment were examined using a Wilcoxon test for assessment of the mean value. Differences in basal values or change in variables between patients in whom OPG levels remained unaltered and those in whom OPG levels decreased, were examined using a Mann-Whitney's U test for assessment of the mean value. Independent association between one dependent and more than two independent variables was assessed by multiple regression analysis. $P$ values of less than 0.05 were considered to be statistically significant.

\section{Results}

\section{Basal levels of serum OPG and clinical variables in the hypothyroid state}

Clinical characteristics of hypothyroid patients and normal controls are shown in Table 1. As mentioned above, all hypothyroid patients exhibited suppression of serum FT4 and FT3 levels with an increase in serum TSH above the normal upper limit. Serum levels of metabolic markers of thyroid function, including serum total cholesterol and LDL-cholesterol, differed significantly between the two groups, but there were no significant differences in age, gender, height, body weight, body mass index (BMI), smoking index 
Table 1 Baseline characteristics of hypothyroid subjects and control subjects. Data expressed as means \pm S.E.

\begin{tabular}{|c|c|c|c|}
\hline & Hypothyroid patients & Normal controls & $P$ \\
\hline Number of subjects & 53 & 53 & \\
\hline Gender (female/male) & $43 / 10$ & $43 / 10$ & \\
\hline Age (years) & $50.9 \pm 2.4$ & $48.2 \pm 2.7$ & ns \\
\hline Height $(\mathrm{cm})$ & $156.2 \pm 1.0$ & $154.6 \pm 1.9$ & ns \\
\hline Body weight $(\mathrm{kg})$ & $56.0 \pm 1.4$ & $53.7 \pm 2.4$ & ns \\
\hline Body mass index $\left(\mathrm{kg} / \mathrm{m}^{2}\right)$ & $22.9 \pm 0.43$ & $22.4 \pm 0.8$ & ns \\
\hline Smoking index & $292 \pm 84$ & $187 \pm 73$ & ns \\
\hline Systolic BP (mmHg) & $118.2 \pm 2.2$ & $121.4 \pm 3.5$ & ns \\
\hline Diastolic BP (mmHg) & $73.3 \pm 1.6$ & $73.3 \pm 2.3$ & ns \\
\hline Mean BP $(\mathrm{mmHg})$ & $95.9 \pm 1.7$ & $97.3 \pm 2.7$ & ns \\
\hline Total cholesterol $(\mathrm{mmol} / \mathrm{l})$ & $5.99 \pm 0.23$ & $5.27 \pm 0.22$ & 0.0374 \\
\hline Triglyceride $(\mathrm{mmol} / \mathrm{l})$ & $1.49 \pm 0.17$ & $1.27 \pm 0.28$ & ns \\
\hline LDL-C (mmol/l) & $3.64 \pm 0.22$ & $2.85 \pm 0.22$ & 0.0212 \\
\hline $\mathrm{HDL}-\mathrm{C}(\mathrm{mmol} / \mathrm{l})$ & $1.55 \pm 0.08$ & $1.8 \pm 0.12$ & ns \\
\hline $\mathrm{OPG}(\mathrm{pmol} / \mathrm{l})$ & $4.514 \pm 0.502$ & $3.720 \pm 0.225$ & 0.0182 \\
\hline FT4 (pmol/l) $(9.01-24.45)$ & $8.82 \pm 0.63$ & $16.2 \pm 0.82$ & $<0.0001$ \\
\hline FT3 $(\mathrm{pmol} / \mathrm{l})(4.00-7.70)$ & $3.41 \pm 0.17$ & $5.56 \pm 0.24$ & $<0.0001$ \\
\hline $\mathrm{TSH}(\mathrm{mIU} / \mathrm{l})(0.4-4.7)$ & $77.3 \pm 10.6$ & $2.2 \pm 0.49$ & $<0.0001$ \\
\hline Creatinine $(\mathrm{mg} / \mathrm{dl})$ & $0.89 \pm 0.12$ & $0.85 \pm 0.14$ & ns \\
\hline vWF activity (\%) & $109.6 \pm 9.4$ & $92.6 \pm 10.6$ & 0.0394 \\
\hline
\end{tabular}

Difference of mean values between two groups were assessed using a Wilcoxon test.

ns, not significant; systolic BP, systolic blood pressure; diastolic BP, diastolic blood pressure; mean BP, mean blood pressure; LDL-C, LDL-cholesterol; HDL-C, HDL-cholesterol; OPG, osteoprotegerin; vWF, von Willebrand factor.

(daily number of cigarettes multiplied by the number of years of smoking), systolic, diastolic, and mean blood pressures, pulse pressure, pulse rate, or serum levels of HDL-cholesterol, triglyceride and creatinine. Serum OPG levels were significantly higher in hypothyroid patients than in normal controls. Mean plasma vWF levels were significantly higher in hypothyroid patients than in normal controls.

\section{Association of basal levels of serum OPG and other parameters in hypothyroid patients before L-T4 replacement therapy}

Table 2 shows the results of multiple regression analysis of the basal levels of various clinical variables to evaluate their association with the basal level of serum OPG in hypothyroid patients. In model 1 , which included vWF, mean blood pressure, BMI and TSH as independent variables, vWF and TSH were found as significant factors that were positively associated with serum OPG. When TSH was replaced by FT3 or FT4, these variables failed to emerge as significant independent determinants of OPG (models 2 and 3). Moreover, in models 4, 5 and 6, which used total cholesterol, LDL-cholesterol and HDL-cholesterol respectively in place of TSH, none of these parameters was found to be significantly associated with serum OPG.

\section{Effect of L-T4 replacement therapy on serum OPG and clinical variables in hypothyroid patients}

In hypothyroid patients, the mean levels of serum OPG decreased significantly during 1 year in a euthyroid state induced by L-T4 replacement therapy to a level comparable to that in normal controls (control value: $3.62 \pm 0.22(n=37)$ vs normal control: $P=0.336)$ (Table 3). L-T4 replacement therapy restored the serum levels of FT4, FT3 and TSH to normal levels and also produced significant decreases in serum total cholesterol and in LDL- and HDL-cholesterol levels. Body weight and BMI were also reduced significantly. No significant change was observed in systolic, diastolic and mean blood pressures, pulse rate, or serum triglyceride and creatinine levels. Serum vWF increased significantly during the year of normalization of thyroid function.

\section{Differences between patients in whom OPG levels remained unaltered and those in whom OPG levels decreased during $L-T 4$ replacement therapy}

We separated the patients into those whose serum OPG levels remained unaltered $(35.1 \%$ of hypothyroid patients) and those whose serum OPG levels decreased (64.9\% of the group) during L-T4 replacement therapy, and the various variables were compared between the two groups of patients. None of the clinical variables listed in Table 1 differed between those patients (data not shown).

\section{Association of baseline characteristics with changes in OPG during $L-T 4$ replacement therapy}

Table 4 shows the results of multiple regression analysis of the basal levels of various clinical variables before L-T4 replacement therapy. These data were used to 
Table 2 Multiple regression analysis to evaluate association of basal levels of serum OPG and other parameters in hypothyroid patients before L-T4 replacement therapy.

\begin{tabular}{|c|c|c|c|c|c|c|}
\hline \multirow[b]{2}{*}{ Independent variables } & \multicolumn{6}{|c|}{ Model } \\
\hline & 1 & 2 & 3 & 4 & 5 & 6 \\
\hline vWF & $0.626^{* *}$ & $0.647^{\star \star}$ & $0.634^{\star *}$ & $0.617^{* *}$ & $0.631^{\star \star}$ & $0.665^{\star *}$ \\
\hline Mean BP & 0.124 & 0.158 & 0.164 & 0.174 & 0.152 & 0.184 \\
\hline BMI & -0.029 & -0.066 & -0.078 & -0.113 & -0.124 & -0.072 \\
\hline TSH & $0.280^{*}$ & & & & & \\
\hline FT3 & & -0.072 & & & & \\
\hline FT4 & & & -0.102 & & & \\
\hline Total cholesterol & & & & -0.133 & & \\
\hline $\begin{array}{l}\text { LDL-C } \\
\text { HDL-C }\end{array}$ & & & & & -0.152 & 0.128 \\
\hline$R^{2}$ & $0.531^{\star *}$ & $0.462^{\star *}$ & $0.468^{* *}$ & $0.481^{\star *}$ & $0.485^{\star *}$ & $0.479^{* *}$ \\
\hline
\end{tabular}

Table 3 Clinical variables in hypothyroid patients before and after one year of normalization of thyroid function by L-T4 replacement therapy. Data are expressed as means \pm S.E.

\begin{tabular}{|c|c|c|c|}
\hline & Before treatment & After 1 year of euthyroid state & $\boldsymbol{P}$ \\
\hline Number of subjects & 37 & 37 & \\
\hline Gender (female/male) & $31 / 6$ & $31 / 6$ & \\
\hline Age (years) & $51.7 \pm 2.9$ & & \\
\hline Body weight (kg) & $54.5 \pm 1.7$ & $53.4 \pm 1.9$ & 0.0067 \\
\hline Body mass index $\left(\mathrm{kg} / \mathrm{m}^{2}\right)$ & $22.2 \pm 0.5$ & $21.7 \pm 0.6$ & 0.0051 \\
\hline Pulse rate $(/ \mathrm{min})$ & $70.2 \pm 1.5$ & $73.0 \pm 2.1$ & ns \\
\hline Systolic BP (mmHg) & $116.0 \pm 2.4$ & $112.5 \pm 2.8$ & ns \\
\hline Diastolic BP (mmHg) & $72.0 \pm 1.7$ & $69.5 \pm 1.6$ & ns \\
\hline Mean BP $(\mathrm{mmHg})$ & $94.0 \pm 1.9$ & $91.0 \pm 2.0$ & ns \\
\hline Total cholesterol (mmol/l) & $5.94 \pm 0.33$ & $4.83 \pm 0.2$ & 0.0006 \\
\hline Triglyceride $(\mathrm{mmol} / \mathrm{l})$ & $1.37 \pm 0.24$ & $1.18 \pm 0.09$ & ns \\
\hline LDL-C (mmol/l) & $3.58 \pm 0.31$ & $2.78 \pm 0.19$ & 0.0079 \\
\hline HDL-C (mmol/li) & $1.72 \pm 0.21$ & $1.51 \pm 0.06$ & 0.0444 \\
\hline $\mathrm{OPG}(\mathrm{pmol} / \mathrm{l})$ & $4.348 \pm 0.505$ & $3.478 \pm 0.262$ & 0.0166 \\
\hline $\mathrm{TSH}(\mathrm{mIU} / \mathrm{l})$ & $75.5 \pm 11.1$ & $2.0 \pm 0.4$ & $<0.0001$ \\
\hline FT4 (pmol/l) & $8.24 \pm 0.67$ & $17.9 \pm 0.86$ & $<0.0001$ \\
\hline FT3 (pmol/l) & $3.25 \pm 0.21$ & $4.52 \pm 0.20$ & $<0.0001$ \\
\hline Creatinine (mg/dl) & $0.86 \pm 0.17$ & $0.84 \pm 0.18$ & ns \\
\hline vWF activity (\%) & $113.3 \pm 12.7$ & $136.8 \pm 12.3$ & 0.0064 \\
\hline
\end{tabular}

Difference of mean values between two groups were assessed using a Wilcoxon test.

ns, not significant; systolic BP, systolic blood pressure; diastolic BP, diastolic blood pressure; mean BP, mean blood pressure; LDL-C, LDL-cholesterol; HDL-C, HDL-cholesterol; OPG, osteoprotegerin; vWF, von Willebrand factor.

evaluate the association of the variables with changes in OPG during L-T4 replacement therapy in hypothyroid patients. In model 1, which included vWF, mean blood pressure, BMI and TSH as independent variables, vWF was the only factor that was significantly and negatively associated with a change in OPG during LT4 replacement therapy. Models 2 and 3, which included FT3 or FT4 in place of TSH, showed no significant association between thyroid hormone levels and changes in OPG. In models 4-6, no association of total cholesterol, LDL-cholesterol or HDL-cholesterol was found with changes in OPG.

\section{Discussion}

In the present study, we demonstrated that the serum level of OPG was significantly higher in hypothyroid patients than in normal controls (Table 1) and that the increased serum OPG returned to a normal level once thyroid function had been normalized, and was sustained within the normal range for at least 1 year. We have recently reported that hypothyroid patients showed increased intima-media thickness in the common carotid artery, and that this arterial thickening was also normalized during sustained normalization of 
Table 4 Multiple regression analysis to evaluate association of change in OPG with other baseline characteristics.

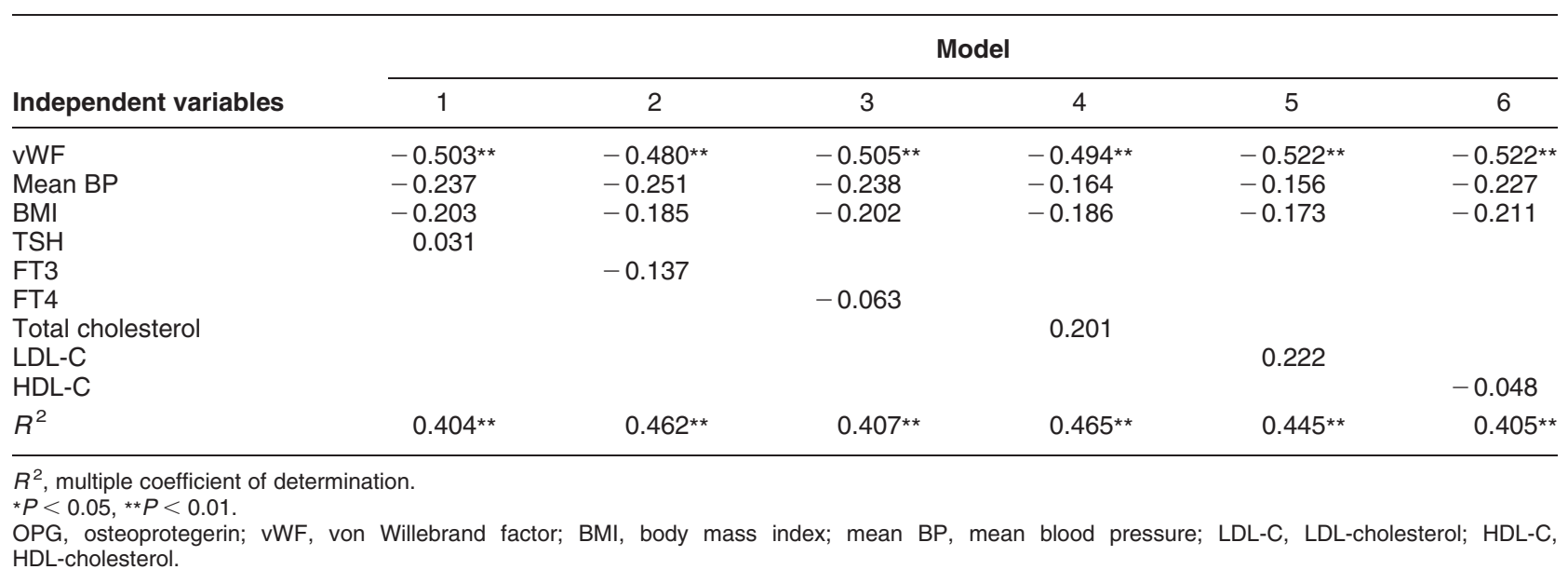

thyroid function by L-T4 replacement therapy (12). These data were in good agreement with a previous report demonstrating that serum osteoprotegerin levels correlate with intima-media thickness in the carotid arteries in healthy postmenopausal women (26). Furthermore, we have observed that the reduction of intima-media thickness in the common carotid artery might have the potential to attenuate thyroid hormone-induced vascular injury, as reflected by an attenuation of the increased response of plasma vWF levels during L-T4 replacement therapy in hypothyroid patients (21).

A recent clinical study suggests that an increase in serum OPG levels is significantly correlated with cardiovascular mortality (6). The increase in serum levels of OPG in hypothyroid patients in the present study was comparable to that seen in a high risk group for the incidence of cardiovascular disease and vascular mortality in the prospective Bruneck Study (6). Furthermore, we have recently reported that the serum OPG level is associated with the presence and severity of coronary artery disease (7), suggesting that serum OPG is a valuable indicator for atherosclerosis and thus a good predictor of cardiovascular events. Supportive of this hypothesis is the recent evidence that OPG is present not only in early atherosclerotic lesions (27) but also in advanced calcified lesions in human atherosclerotic plaques in the carotid artery (5). Taking these data into consideration, it was suggested that an increase in serum OPG might also be a good indicator for increased arterial thickening in hypothyroid patients.

OPG is known to exert an inhibitory effect on arterial calcification and osteoclastogenesis $(1,4)$, although the molecular mechanism underlying the role of OPG in the progression of atherosclerosis is not fully understood. Our findings showed a significant and positive association between baseline OPG and baseline vWF or TSH in hypothyroid patients (Table 2). Since vWF is a reliable parameter for endothelial vascular injury
(17-21), particularly in hypothyroid patients (21), these findings support the notion that an increase in serum OPG levels is also a reliable marker for vascular injury in hypothyroidism $(8,9)$. However, the baseline level of vWF, but not that of TSH, was significantly and independently negatively associated with the change in serum OPG during 1 year of euthyroidism (Table 4). These findings suggest that hypothyroid patients in whom serum vWF levels had been higher before L-T4 replacement therapy exhibited a greater decrease in serum OPG levels during treatment, supporting the association of an increase in serum OPG levels in hypothyroidism with vascular injury, rather than with the hypothyroid state itself. The limitation of the present study is that the multiple regression models shown in Table 2 explain only 46.2 to $57.1 \%$ of the baseline OPG levels, indicating the possible presence of other regulatory factors that lead to elevation of serum OPG levels in hypothyroid patients. Hence, we examined the possible involvement of other established risk factors for atherosclerosis in the increase in serum OPG levels in hypothyroidism. Unfortunately, no data are available for the decrease in serum OPG levels during treatment for hyperlipidemia or hypertension. Although hypothyroidism induces hyperlipidemia (13), no significant association was found between serum OPG levels and the baseline level of serum lipids or changes in the serum lipid level during L-T4 replacement therapy (Tables 2 and 4 ), negating the possible involvement of hyperlipidemia-induced vascular injury in the increase in serum OPG levels. Although thyroid hormone causes vascular injury via multiple mechanisms (20, 28-30), including its effect on the hemodynamic state, no association was found between serum OPG and hemodynamic factors such as mean blood pressure (Tables 2 and 4), systolic and diastolic blood pressure, and pulse rate in the present study. These findings clearly negated the possibility that alterations in serum lipid levels and 
the hemodynamic states induced by hypothyroidism might be involved in the regulation of OPG in hypothyroid patients.

The clearance of OPG or its metabolism was reported to be under the influence of renal function (31) and creatinine clearance could be influenced by thyroid hormone (32). Although we have no data about the creatinine clearance, serum creatinine did not change significantly before and after L-T4 replacement therapy (Table 3). These data may negate the possibility, although not completely, that decreased clearance of OPG from serum in the hypothyroid state might increase serum OPG levels.

In summary, this is the first report demonstrating that hypothyroid patients exhibit a significant increase in serum OPG levels compared with normal controls, and that normalization of thyroid function for 1 year by L-T4 replacement therapy significantly decreases serum OPG to levels comparable to those in normal controls. It was further suggested that the increase in serum OPG levels in hypothyroid patients is independently associated with vascular injury, as reflected by increased plasma vWF levels, but not with the severity of hypothyroidism.

\section{References}

1 Wooden S, Bennett L, Boone T, Shimamoto G, DeRose M, Elliott R, Colombero A, Tan HL, Trail G, Sullivan J, Davy E, Bucay N, Renshaw-Gegg L, Hughes TM, Hill D, Pattison W, Campbell P \& Boyle WJ. Osteoprotegerin: a novel secreted protein involved in the regulation of bone density. Cell $1997 \mathbf{8 9} 309-319$.

2 Hofbauer LC, Shui C, Riggs BL, Dunstan CR, Spelsberg TC, O'Brien T \& Khosla S. Effects of immunosuppressants on receptor activator of N-kappaB ligand and osteoprotegerin production by human osteoblastic and coronary artery smooth muscle cells. Biochemical and Biophysical Research Communications 2001280 334-339.

3 Yun TJ, Chaudhary PM, Shu GL, Frazer JK, Ewings MK, Schwartz SM, Pascual V, Hood LE \& Clark EA 3. OPG/FDCR-1, a TNF receptor family member, is expressed in lymphoid cells and is up-regulated by ligating CD40. Journal of Immunology $19981616113-6121$.

4 Bucay N, Sarosi I, Dunstan CR, Morony S, Tarpley J, Capparelli C, Scully S, Tan HL, Xu W, Lacey DL, Boyle WJ \& Simonet WS. Osteoprotegerin-deficient mice develop early onset osteoporosis and arterial calcification. Genes and Development $1998 \quad 12$ 1260-1268.

5 Dhore CR, Cleutjens JP, Lutgens E, Cleutjens KB, Geusens PP, Kitslaar PJ, Tordoir JH, Spronk HM, Vermeer C \& Daemen MJ. Differential expression of bone matrix regulatory proteins in human atherosclerotic plaques. Arteriosclerosis, Thrombosis and Vascular Biology 200421 1998-2003.

6 Kiechl S, Schett G, Wenning G, Redlich K, Oberhollenzer M, Mayr A, Santer P, Smolen J, Poewe W \& Willeit J. Osteoprotegerin is a risk factor for progressive atherosclerosis and cardiovascular disease. Circulation 2004109 2175-2180.

7 Jono S, Ikari Y, Shioi A, Mori K, Miki T, Hara K \& Nishizawa Y. Serum osteoprotegerin levels are associated with the presence and severity of coronary artery disease. Circulation $2002 \mathbf{1 0 6}$ $1192-1194$.

8 Auer J, Berent R, Weber T, Lassnig E \& Eber B. Thyroid function is associated with the presence and severity of coronary atherosclerosis. Clinical Cardiology 200326 569-573.
9 Cappola AR \& Ladenson PW. Hypothyroidism and atherosclerosis. Journal of Clinical Endocrinology and Metabolism $2003 \mathbf{8 8}$ $2438-2444$.

10 Lekakis JP, Papamichael CM, Cimponeriu AT, Stamatclopoulos KS, Papaioannou TG, Kanakakis J, Alevizaki MK, Papapanagiotou A, Kalofoutis AT \& Stamatclopoulos SF. Atherosclerotic changes of extracoronary arteries are associated with the extent of coronary atherosclerosis. American Journal of Cardiology $2000 \quad \mathbf{8 5}$ 949-952.

11 Simons PC, Algra A, Bots ML, Grobbee DE \& van der Graaf Y. Common carotid intima-media thickness and arterial stiffness: indicators of cardiovascular risk in high-risk patients. The SMART Study (Second Manifestations of Arterial disease). Circulation $1999100951-957$.

12 Nagasaki T, Inaba M, Henmi Y, Kumeda Y, Ueda M, Tahara H, Sugiguchi S, Fujiwara S, Emoto M, Ishimura E, Onoda N, Ishikawa T \& Nishizawa Y. Decrease in carotid intima-media thickness in hypothyroid patients after normalization of thyroid function. Clinical Endocrinology $200359607-612$.

13 Becerra A, Bellido D, Luengo A, Piedrola G \& De Luis DA. Lipoprotein(a) and other lipoproteins in hypothyroid patients before and after thyroid replacement therapy. Clinical Nutrition 199918 319-322.

14 Muller B, Tsakiris DA, Roth CB, Guglielmetti M, Staub JJ \& Marbet GA. Haemostatic profile in hypothyroidism as potential risk factor for vascular or thrombotic disease. European Journal of Clinical Investigation 200131 131-137.

15 Lekakis JP, Papamichael CM, Alevizaki MK, Piperingos G, Marafelia P, Mantzos J, Stamatelopoulos S \& Koutras DA. Flow- mediated, endothelium-dependent vasodilation is impaired in subjects with hypothyroidism, borderline hypothyroidism, and high-normal serum thyrotropin (TSH) values. Thyroid 1997 7 411-414.

16 Berenson GS \& Srinivasan SR. Emergence of obesity and cardiovascular risk for coronary artery disease: the Bogalusa Heart Study. Preventive Cardiology 2001 4 116-121.

17 Ware LB, Eisner MD, Thompson BT, Parsons P \& Matthay MA. The acute respiratory distress syndrome network significance of von Willebrand factor in septic and non-septic patients with acute lung injury. American Journal of Respiratory and Critical Care Medicine $2004170766-772$.

18 Doshi SN, Moat SJ, Lewis MJ, McDowell IF, Giddings JC \& Goodfellow J. Short-term high-dose folic acid does not alter markers of endothelial cell damage in patients with coronary heart disease. International Journal of Cardiology $2004 \mathbf{9 4}$ 203-207.

19 Makin AJ, Blann AD, Chung NA, Silverman SH \& Lip GY. Assessment of endothelial damage in atherosclerotic vascular disease by quantification of circulating endothelial cells. Relationship with von Willerbrand factor and tissue factor. European Heart Journal $200425371-376$.

20 Kurioka Y, Inaba M, Kawagishi T, Emoto M, Kumeda Y \& Nishizawa Y. Increased retinal blood flow in patients with Graves' disease: influence of thyroid function and ophthalmopathy. European Journal of Endocrinology 2000144 1-9.

21 Nagasaki T, Inaba M, Henmi Y, Kumeda Y, Ueda M, Tahara H, Ishimura E, Onoda N, Ishikawa T \& Nishizawa Y. Change in von Willebrand factor and carotid intima-media thickness in hypothyroid patients with normal thyroid function after levothyroxine replacement therapy. European Journal of Endocrinology $2004150125-131$.

22 Nacfralane DE, Stibbe J, Kirby EP, Zucker MB, Grant RA \& McPherson J. A method for assaying von Willebrand factor (Ristocetin cofactor). Thrombosis et Diathesis Haemorrhagica 197534 306-308.

23 Friedewald WT, Levy RI \& Fredrickson DS. Estimation of the concentration of low-density lipoprotein in plasma without use of preparative ultracentrifuge. Clinical Chemistry 197218499.

24 Christofides ND \& Sheehan CD. Enhanced chemiluminescence labeled-antibody immunoassay (Amerlite-MAB) for free 
thyroxine: design, development, and technical validation. Clinical Chemistry 1995 41/1 17-23.

25 Yano K, Tsuda E, Washida N, Kobayashi F, Goto M, Harada A, Ikeda K, Higashio K \& Yamada Y. Immunological characterization of circulating osteoprotegerin/osteoclastogenesis inhibitory factor: increased serum concentrations in postmenopausal women with osteoporosis. Journal of Bone and Mineral Research 199914 518-527.

26 Erdogan B, Aslan E, Bagis T, Gokcel A, Erkanli S, Bavbek M \& Altinors N. Intima-media thickness of the carotid arteries is related to serum osteoprotegerin levels in healthy postmenopausal women. Neurological Research 200426 658-661.

27 Golledge J, McCann M, Mangan S, Lam A \& Karan M. Osteoprotegerin and osteopontin are expressed at high concentrations within symptomatic carotid atherosclerosis. Stroke 200435 1636-1641.

28 Klein I. Thyroid hormone and the cardiovascular system. American Journal of Medicine $1990 \mathbf{8 8} 631-637$.

29 Saito I, Ito K \& Saruta T. The effect of age on blood pressure in hyperthyroidism. Journal of the American Geriatrics Society 1985 $3319-22$.
30 Inaba M, Henmi Y, Kumeda Y, Ueda M, Nagata M, Emoto M, Ishikawa T, Ishimura E \& Nishizawa Y. Increased stiffness in common carotid artery in hyperthyroid Graves' disease patients. Biomedicine and Pharmacotherapy $200256241-246$.

31 Sato T, Tominaga Y, Iwasaki Y, Kazama JJ, Shigematsu T, Inagaki H, Watanabe I, Katayama A, Haba T, Uchida K \& Fukagawa M. Osteoprotegerin levels before and after renal transplantation. American Journal of Kidney Diseases 200138 175-177.

32 Karanikas G, Schutz M, Szabo M, Becherer A, Wiesner K, Dudczak R \& Kletter K. Isotopic renal function studies in severe hypothyroidism and after thyroid hormone replacement therapy. American Journal of Nephrology 200424 41-45.

Received 7 October 2004

Accepted 14 December 2004 